\title{
Different Evolutionary Stages in the Massive Star-forming Complex W3 Main
}

\author{
Yuan Wang ${ }^{1,2}$, Henrik Beuther ${ }^{2}$, Qizhou Zhang ${ }^{3}$, Arjan Bik ${ }^{2}$, Javier \\ A. Rodón ${ }^{4}$, Zhibo Jiang ${ }^{1}$, and Cassandra Fallscheer ${ }^{5}$ \\ ${ }^{1}$ Purple Mountain Observatory, 2 West Beijing Road, Nanjing 210008, China \\ email: ywang@pmo.ac.cn \\ ${ }^{2}$ MPIA, ${ }^{3}$ Harvard-Smithsonian CfA, ${ }^{4}$ ESO, Chile, ${ }^{5}$ University of Victoria
}

\begin{abstract}
We observed with the Submillimeter Array and IRAM $30 \mathrm{~m}$ telescope three highmass star-forming regions in different evolutionary stages in the W3 high-mass star formation complex. These regions, i.e. W3 SMS1 (W3 IRS5), SMS2 (W3 IRS4) and SMS3, are located within the same large-scale environment, which allows us to study rotation and outflows as well as chemical properties in an evolutionary sense. While we find multiple mm continuum sources toward all regions, these three subregions exhibit different dynamical and chemical properties, which indicates that they are in different evolutionary stages. Even within each sub-region, massive cores of different ages are found, e.g. in SMS2, sub-sources from the most evolved UCHII region to potential starless cores exist within 30000 AU (left panel, Fig. 1). Outflows and rotational structures are found in SMS1 and SMS2. Evidence for interactions between the molecular cloud and the HII regions is found in the ${ }^{13} \mathrm{CO}$ channel maps (right panel, Fig. 1), which may indicate triggered star formation.
\end{abstract}

Keywords. stars: formation — ISM: molecules — ISM: individual objects (W3)
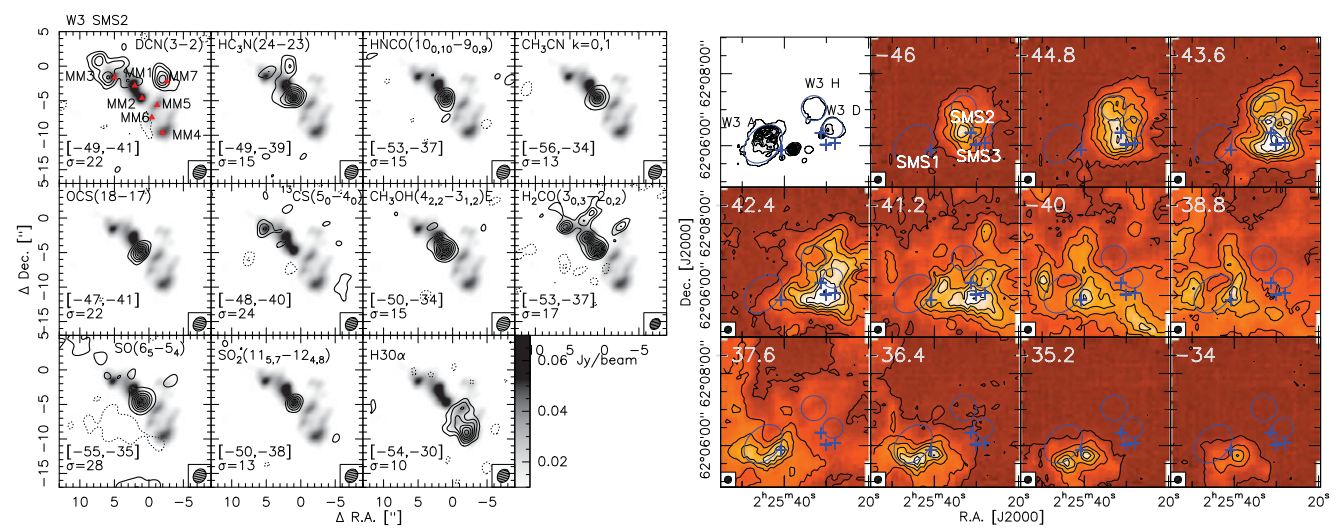

Figure 1. Left: W3 SMS2 molecular line integrated intensity images overlaid on the SMA dust continuum emission in the background. Right: The top left panel shows the $6 \mathrm{~cm}$ VLA continuum image (Tieftrunk et al. 1997), and the rest of the panels show the single-dish ${ }^{13} \mathrm{CO}(2-1)$ channel map with the spectral resolution of $1.2 \mathrm{~km} \mathrm{~s}^{-1}$. The crosses mark the position of the SMA continuum sources SMS1-MM1, SMS2-MM2, SMS3-MM1, and SMS3-MM2.

\section{References}

Tieftrunk, A. R., Gaume, R. A., Claussen, M. J., Wilson, T. L., \& Johnston, K. J. 1997, A\&A, 318, 931 